University of Wollongong

Research Online

Australian Institute for Innovative Materials -

Papers

Australian Institute for Innovative Materials

$1-1-2019$

\title{
Self-Healing Electrode with High Electrical Conductivity and Mechanical Strength for Artificial Electronic Skin
}

\author{
Hyeon Jun Sim \\ Hanyang University \\ Hyunsoo Kim \\ Hanyang University \\ Yongwoo Jang \\ Hanyang University \\ Geoffrey M. Spinks \\ University of Wollongong, gspinks@uow.edu.au \\ Sanjeev Gambhir \\ University of Wollongong, sanjeev@uow.edu.au
}

See next page for additional authors

Follow this and additional works at: https://ro.uow.edu.au/aiimpapers

Part of the Engineering Commons, and the Physical Sciences and Mathematics Commons

\footnotetext{
Research Online is the open access institutional repository for the University of Wollongong. For further information
} contact the UOW Library: research-pubs@uow.edu.au 


\title{
Self-Healing Electrode with High Electrical Conductivity and Mechanical Strength for Artificial Electronic Skin
}

\author{
Abstract \\ A self-healing electrode is an electrical conductor that can repair internal damage by itself, similar to \\ human skin. Since self-healing electrodes are based on polymers and hydrogels, these components are \\ still limited by low electrical conductivity and mechanical strength. In this study, we designed an \\ electrically conductive, mechanically strong, and printable self-healing electrode using liquid crystal \\ graphene oxide (LCGO) and silver nanowires (AgNWs). The conductive ink was easily prepared by simply \\ mixing LCGO and AgNWs solutions. The ultrathin ( $3 \mu \mathrm{m}$ thick) electrode can be printed in various shapes, \\ such as a butterfly, in a freestanding state. The maximum conductivity and strength of the LCGO/AgNW \\ composite were $17 » 800 \mathrm{~S} / \mathrm{cm}$ and $4.2 \mathrm{MPa}$, respectively; these values are 24 and 4 times higher, \\ respectively, than those of a previously developed self-healing electrode. The LCGO/AgNW composite \\ self-healed internal damage in ambient conditions with moisture and consequently recovered $96.8 \%$ \\ electrical conductivity and $95 \%$ mechanical toughness compared with the undamaged state. The \\ electrical properties of the composite exhibited metallic tendencies. Therefore, these results suggest that \\ the composite can be used as an artificial electronic skin that detects environmental conditions, such as \\ compression and temperature. This self-healing artificial electronic skin could be applied to human \\ condition monitoring and robotic sensing systems. \\ Disciplines \\ Engineering | Physical Sciences and Mathematics

\section{Publication Details} \\ Sim, H. Jun., Kim, H., Jang, Y., Spinks, G. M., Gambhir, S., Officer, D. L., Wallace, G. G. \& Kim, S. Jeong. \\ (2019). Self-Healing Electrode with High Electrical Conductivity and Mechanical Strength for Artificial \\ Electronic Skin. ACS Applied Materials and Interfaces, Online First 1-8.
}

\section{Authors}

Hyeon Jun Sim, Hyunsoo Kim, Yongwoo Jang, Geoffrey M. Spinks, Sanjeev Gambhir, David L. Officer, Gordon G. Wallace, and Seon Jeong Kim 


\section{A Printable Self-Healing Electrode with High Electrical Conductivity and Mechanical Strength for Smart Electronics}

Hyeon Jun Sim, Hyunsoo Kim, Yongwoo Jang, Geoffrey M. Spinks, Sanjeev Gambhir, David L. Officer, Gordon G. Wallace, and Seon Jeong Kim*

1. H. J. Sim, H. Kim, Y. Jang, Prof. S. J. Kim

Center for Self-Powered Actuation, Department of Biomedical Engineering, Hanyang University, Seoul 04763, Korea

2. G. M. Spinks, S. Gambhir, D. L. Officer, G. G. Wallace

Intelligent Polymer Research Institute, ARC Centre of Excellence for Electromaterials Science, AIIM Facility, Innovation Campus, University of Wollongong, North Wollongong, NSW 2522, Australia

*To whom correspondence should be addressed. E-mail: sjk@ hanyang.ac.kr 


\section{ABSTRACT:}

The self-healing electrode is an electrical conductor that can recover internal damage by itself like human skin. Since self-healing electrodes are based on polymers and hydrogels, it is still limited in low electrical conductivity and mechanical strength. Herein, we designed an electrically conductive, mechanically strong and printable self-healing electrode using liquid crystal graphene oxide (LCGO) and silver nanowires (AgNWs). The conductive ink was easily prepared by simply mixing LCGO and AgNWs solutions. The ultrathin ( $3 \mu \mathrm{m}$ thick) electrode can be printed in various shapes such as a butterfly in a freestanding state. The maximum conductivity and strength of the LCGO/AgNWs composite were 17,800 S/cm and 4.2 MPa, values that are respectively 24 and 4 times higher than for a previous self-healing electrode. The LCGO/AgNWs composite has selfhealed internal damage in ambient condition with moisture, and consequently recovered $96.8 \%$ of its electrical conductivity and $95 \%$ of its mechanical toughness compared to undamaged state. The electrical properties of the composite exhibited a metallic tendency. Therefore, these results suggest that the composite can be used as an artificial electronic skin that detects environmental conditions such as compression and temperature. The self-healing artificial electronic skin could be applied to human condition monitoring and robotic sensing systems.

KEYWORDS: Self-healing, Conductive, Strength, Electrode, Electronic skin 
With the increasing need for wearable electronics, wearable electrodes that are flexible, lightweight, durable and safe to wear have been developed. ${ }^{1-5}$ The wearable electrode is used for industrial applications such as ubiquitous healthcare, electronic skin, robotics, safety equipment and environmental monitoring systems. To date, the self-healing electrode which is self-repair property like human skin is required to enhance the durability. ${ }^{6-18}$ The remarkable property allows the device to function with sustainable performance, despite the electrode being subject to damage such as scratching, cracking and mechanical cuts. In particular, electronic skin for a human monitoring system based on a self-healing electrode received much attention after the electronic skin was repeatedly bent and directly exposed to impact and compression in daily motion. Also, the self-healing electrode-based electronic skin can be used in extreme environment which is in the difficult condition to repair.

Huynh et al. designed a self-healable composite with self-healable polyurethane and silver. ${ }^{6}$ The composite was self-healed in ambient condition and was able to recover nearly $100 \%$ of its electrical properties. Moreover, Darabi et al. developed a mechanically and electrically selfhealing hydrogel with poly(acryl acid), polypyrrole, chitosan and ferric ions. ${ }^{7}$ The conductive hydrogel has bulk conductivity, electrical and mechanical self-healing property and pressure sensitivity.

However, previous self-healing electrodes have developed based on a polymer and hydrogel, and thus it shows a relatively low conductivity (below $700 \mathrm{~S} / \mathrm{cm}$ ). ${ }^{6,17}$ The low electrical conductivity induced energy loss and low efficiency. In addition, most self-healing electrode had a relatively low strength (below $1 \mathrm{MPa})^{7,12,16}$ due to the inherent mechanical property of polymer and hydrogel. The low mechanical property means that the electrode could be easily deformed and restricted in daily applications. 
Here, we designed an electrically conductive, mechanically strong and printable self-healing electrode using liquid crystal graphene oxide (LCGO) and silver nanowires (AgNWs). The electrode was fabricated by printing a conductive ink in which an LCGO solution was mixed with an AgNWs solution. The maximum conductivity and strength of the LCGO/AgNWs composite were $17800 \mathrm{~S} / \mathrm{cm}$ and $4.2 \mathrm{MPa}$, i.e., 24 and 4 times higher than the previous self-healing electrode. The LCGO/AgNWs electrode self-healed under ambient condition with moisture and was able of recovering $96.8 \%$ of its electrical conductivity and $95 \%$ of its mechanical toughness. As its application, we fabricated a self-healing artificial electronic skin, and showed to detect environmental conditions such as compression and temperature. This result suggest that the selfhealing artificial electronic skin could be applied to human condition monitoring and robotic sensing systems.

A schematic illustration showing the fabrication process of the printable self-healing electrode is shown in Fig. 1a. To make a self-healable electrode, a $1 \mathrm{wt} \%$ LCGO with a lateral size of up to $100 \mu \mathrm{m}$ dispersed in a water solution was prepared. ${ }^{19}$

Next, AgNWs dispersed in an ethanol solution were added to the LCGO solution to make conductive ink. When the AgNWs solution was added to the LCGO solution, the solution was homogeneously mixed without aggregation. A conductive ink was formed as a gel-like mud and a 3D cubic structure was made with the dimensions of $1 \mathrm{~cm}$ length, $1 \mathrm{~cm}$ width and $3 \mathrm{~mm}$ depth (Fig. S1). LCGO and AgNWs were chosen as a self-healing electrode material based on the following considerations. (1) LCGO could bond with AgNWs by a functional group. (2) The largescale graphene oxide-based composite has a high tensile strength because of hydrogen bonding. (3) AgNWs are highly conductive materials and (4) the entire process is inexpensive and solutionprocessable. ${ }^{20}$ 
Finally, the conductive ink was printed on the substrates and dried in ambient condition. On the Teflon substrate, the printed electrode was easily detached from the substrate (Fig. 1b). The complete freestanding film was easily bent without distortion because of its good mechanical property.

The surface morphology of the printed LCGO/AgNWs composite is shown in Fig. 1c. The AgNWs were randomly distributed on the large-scale wrinkled graphene oxide. The electrical network was formed as the AgNWs were in contact with each other. The wrinkled morphology of the LCGO could be explained by the formation of hydrogen bonds, with an functional group such as hydroxyl, epoxide, ketone and carboxyl. It is believed that the functional group causes full delamination of the LCGO monolayer sheet in aqueous solution. The uniform film was ultrathin, about $3 \mu \mathrm{m}$ thick (Fig. 1d). The solution-based fabrication process could save fabrication cost and time, and could be applied to microscale printing. In the magnified scanning electron microscope (SEM) image, the LCGO/AgNWs composite was formed as a layered structure in which the LCGO and AgNWs were alternately stacked (Fig. 1e). The layered structure indicates that the conductive ink was homogeneously mixed without aggregation and AgNWs forged a bond with LCGO.

The electrical conductivity of the LCGO/AgNWs composite vs. weight of AgNWs is shown in Fig. 1f. Five samples for each weight ratio (25 in total) were measured, and the average conductivities were plotted. The conductivity in the LCGO/AgNWs composite increased with the weight of AgNWs from $0.011 \mathrm{~S} / \mathrm{cm}$ at $20 \mathrm{wt} \%$ to $17000 \mathrm{~S} / \mathrm{cm}$ at $80 \mathrm{wt} \%$. The conductivities for the $20 \mathrm{wt} \%$ and $80 \mathrm{wt} \% \mathrm{LCGO} / \mathrm{AgNW}$ composites were $1.2 \times 10^{3}$ and $1.9 \times 10^{9}$ times higher than the conductivity of graphene oxide film $(0.000009 \mathrm{~S} / \mathrm{cm})$, respectively.

In the previous report, self-healing polyurethane was used with silver particles to achieve the self-healing property. However, the polymer can prevent the electrical pathway and take up a large 
volume in the composite, resulting in a relatively low electrical conductivity $(700 \mathrm{~S} / \mathrm{cm}){ }^{6}$ Compared with the literature ${ }^{6}$, the electrical conductivity of the LCGO/AgNWs composite was 24 times higher than the previous self-healing electrode.

The high electrical conductivity of the LCGO/AgNWs composite originated not only from the high conductivity of AgNWs but also from the small volume of the composite. In the case of the LCGO/AgNWs composite, the distance between each LCGO/AgNWs was close after evaporation, as a result, the total volume was small and the AgNWs were electrically connected to each other.

The high conductivity makes the composite suitable for use as an electrode in an electronic device. The highly conductive electrode could enhance the efficiency of the electric device and reduce the risk of side effects such as heat dissipation.

For a real application, mechanical strength is required to resist external stimuli. The stressstrain curve of the LCGO/AgNWs composite to analyze the mechanical property is shown in Fig. 1g. The ultimate tensile stress (UTS) for the $20 \mathrm{wt} \%$ and $80 \mathrm{wt} \%$ electrodes were about $26.1 \mathrm{MPa}$ and 4.2 MPa, respectively.

The high strength originated from the functional group of LCGO. The hydrogen bonds are formed by the functional group, and as the result, the graphene layers are strongly bonded to each other. When the weight of AgNWs increases in the LCGO/AgNWs composite, the AgNWs bond to the functional group of $\mathrm{LCGO}^{20}$ and the total bonding force decreased. Therefore, the mechanical strength of the LCGO/AgNWs composite decreased with increasing weight of AgNWs in the composite.

As the reported self-healing electrode was based on a polymer and hydrogel, the previous selfhealing electrode has a relatively low strength (below $1 \mathrm{MPa}$ ) ${ }^{7,12,16}$ In daily life, a wearable device 
is directly exposed to external stimuli such as impact, compression, scratching, cracking and mechanical cuts and low mechanical properties are a limitation for real applications.

The strength of the $80 \mathrm{wt} \% \mathrm{LCGO} / \mathrm{AgNW}$ s composite was 4 times higher than the previous selfhealing electrode. ${ }^{7,12,16}$ The electrode could resist the external stimuli and was able to operate continuously in a real application. As the $80 \mathrm{wt} \% \mathrm{LCGO} / \mathrm{AgNWs}$ composite had high conductivity and strength, it was used for the following experiment as the LCGO/AgNWs electrode.

The self-healing property is a crucial issue for a sustainable and semipermanent system. The self-healing performance of the LCGO/AgNWs electrode is shown in Fig. 2 a-c. The butterflyshaped electrode was printed with a syringe. After that, the electrode was fist-crumpled and damaged. After moisture was sprayed on the damaged point, the damaged point recovered to the initial state.

The mechanism of the healing process is explained (Fig. S2). After spraying moisture on the damaged point, the wet LCGO sheets were swollen by absorbing water, resulting in partially breaking the hydrogen bonding interaction. When the swollen LCGO sheets came into contact with other LCGO sheets, the other sheets were filled with water by inter-diffusion and capillary force. ${ }^{21}$ After the water evaporated, the distance between the LCGO sheets was physically close, resulting in the formation of new hydrogen bonding and reconnecting with each other.

The SEM images show the morphology and structure change of the LCGO/AgNWs electrode during the self-healing process (Fig. $2 \mathrm{~d}-\mathrm{f}$ ). When the electrode was damaged, the electrical network of the AgNWs was disconnected. After spraying with moisture, the LCGO/AgNWs electrode was swollen by water and filled the damaged space. After water evaporation, the swollen electrode in water bonded with the surrounding composite and self-healing was successfully achieved. 
To investigate the electrical healing of the LCGO/AgNWs electrode, the electrode was completely bifurcated using a blade and the two fractured surfaces were brought together. The voltage-current curve of the $\mathrm{LCGO} / \mathrm{AgNW}$ s electrode before and after the healing process is shown in Fig. 3a. The electrode displayed a linear behavior and with the non-hysteretic currentvoltage characteristics a good electrical conductor was expected. The conductivity of silver is much higher than that of graphene oxide and the AgNWs mainly affect the electrical conductivity of the electrode.

When the electrode was completely cut, an open circuit was formed and the current was close to zero. After the healing process, the curve of the healed electrode was nearly the same as the original electrode, for which the electrical healing efficiency (the proportion of conductivity restored relative to the original conductivity) was about $96.9 \%$. The electrical resistance varies with time during the healing process. As the edges of the electrode were brought into contact, the resistance immediately dropped. The contact induced the restoration of the electrical property and the electrical healing efficiency reached as high as $89 \%$. After several minutes, the conductivity returns close to its initial value.

Repeated cuts at the same location were healed, indicating repeatable restoration of the electrical performance in an LCGO/AgNWs electrode (Fig. 3c). The repeatable restoration of electrical property was demonstrated above the electrical healing efficiency of $96 \%$ in our selfhealing electronic composite.

Regarding the mechanical healing of the LCGO/AgNWs electrode, the stress-strain curves of the original and healed composites are shown in Fig. 3d. The tensile strain and strength of the original composite were $1.32 \%$ and $4.18 \mathrm{MPa}$, respectively. After the healing process, the tensile strain and strength of the healed electrode were $1.38 \%$ and 3.97 MPA, respectively. The 
mechanical healing efficiency (the proportion of toughness restored relative to the original toughness) recovered was about $95 \%$.

Fig. 3e shows that the LCGO/AgNWs electrode can be used in an electronic circuit for devices such as light-emitting diodes (LEDs). The conductive composite was connected with a copper electrode, a blue LED and a $3 \mathrm{~V}$ commercial battery. When the electric circuit was connected, the blue LED was on because the conductivity of the LCGO/AgNWs electrode was enough to light it. When the composite was bifurcated, the resistance enormously increases and the LED was off. After the healing process, the blue LED was on again as the conductivity of the healed electrode was similar to the original composite.

The electrical stability of the electrode to endure external stimuli such as bending and twisting was crucial. For a wearable system, the stability of the LCGO/AgNWs composite electrode is shown (Fig. S3-4). During bending and twisting, the resistance was nearly identical. Moreover, the electrode was stable with repeated bending (Fig. S5). The resistance was constant in repeated cycles because the AgNWs network structure was highly stable to mechanical deformation. After the healing process, the resistance was constant during repeated cycles. The results indicate that the healed point recovered well without distortion.

The LCGO/AgNWs electrode is useful for application as artificial electronic skin (Fig. 4a). A self-healing tactile sensor was fabricated by printing LCGO/AgNWs conductive ink on the dielectric. The ink was separately printed on both sides of a rectangular silicone rubber dielectric. The capacitance of the tactile sensor dependent on stress in the thickness direction was measured as shown in Fig. 4b. When the pressure was applied perpendicular to the electrode stack (from 0 to $9.8 \mathrm{kPa}$ ), the dielectric thickness decreased and the electrode area increased. Therefore, the 
capacitance increased from 7.4 to $8.2 \mathrm{pF}$. Stable, reversible capacitive response cycles were obtained during repeated compression and releasing (Fig. 4c).

The LCGO/AgNWs electrode can be used not only as a wearable electrode of a tactile sensor but also as a resistance temperature detector (RTD) (Fig. 4d).

The wearable RTD was fabricated by printing LCGO/AgNWs ink on a sticker that is a polyethylene terephthalate (PET) film coated with a sticky substance only on one side. The resistance of the LCGO/AgNWs electrode linearly increased and decreased with temperature regardless of the heating and cooling state. The resistance ratio increased to 1.25 at $100{ }^{\circ} \mathrm{C}$ compared with the resistance at $20^{\circ} \mathrm{C}$. The LCGO/AgNWs-based RTD has stability, accuracy and repeatability because the composite has metallic properties because of the AgNWs. Wearable RTDs are continuously able to measure temperature and ambient temperature change in real time (Fig. 4e). When the temperature was immediately changed, the resistance was changed in real time. The fast response time originated from the high thermal conductivity of the AgNWs and the microscale thickness. Because the fabrication process was based on the solution printing method, the process is simple and an artistically designed sensor could be easily fabricated.

\section{Conclusion}

The present study showed that the conductivity of the LCGO/AgNWs composite increases from $0.011 \mathrm{~S} / \mathrm{cm}$ at $20 \mathrm{wt} \%$ to $17,000 \mathrm{~S} / \mathrm{cm}$ at $80 \mathrm{wt} \%$ dependent on the weight of AgNWs. Consequently, the conductivities of the $20 \mathrm{wt} \%$ and $80 \mathrm{wt} \%$ electrodes were $1.2 \times 10^{3}$ and $1.9 \times$ $10^{9}$ times higher than that of the graphene oxide film $(0.000009 \mathrm{~S} / \mathrm{cm})$, respectively. The UTS and Young's modulus decreased with the weight of AgNWs and the UTS of the $20 \mathrm{wt} \%$ and $80 \mathrm{wt} \%$ electrode was about 26.1 and 4.2 MPa, respectively. Although the UTS of the $80 \mathrm{wt} \%$ composite 
was much lower than the graphene-oxide-only electrode, the UTS was 4 times higher than that of the previous self-healing electrode of below $1 \mathrm{MPa}$ because the reported self-healing electrode was based on polymer and hydrogel. The LCGO/AgNWs electrode has self-healed under ambient condition with moisture and was able to recover $96.8 \%$ of the electrical conductivity and $95 \%$ of its strength. The LCGO/AgNWs electrode can be easily attached to human skin like a tattoo and the LCGO/AgNWs can act as a resistance temperature detector as the electrode has stability, accuracy and repeatability.

\section{Experimental section}

\section{Fabrication process of LCGO/AgNWs ink}

$1 \mathrm{wt} \%$ LCGO with a lateral size of up to $100 \mu \mathrm{m}$ dispersed in water solution was prepared. ${ }^{[19]}$ After that, $1 \mathrm{wt} \%$ AgNWs dispersed in ethanol solution (S27E-KNS7C4, Nanopyxis, Korea) was added to the LCGO solution to make conductive ink. The conductive ink was homogeneously mixed by a stirrer (pc-420d, Corning, New york, USA) for $1 \mathrm{~h}$. The conductive ink was poured by a $5 \mathrm{ml}$ syringe and printed on the Teflon substrate. The printed ink was dried in ambient condition for 24 h.

\section{Characterization}

The morphology of the LCGO/AgNws composite was determined by SEM (SEM-S4700 microscope, Hitachi, Japan). The conductivity of each composite was measured by a four-point probe sheet resistance measurement system (CMT-100s, Advanced Instrument Technology, Georgia, USA). The voltage-current curves were examined using a source meter (2635A, Keithley, Ohio, USA). The resistance of the rectangle shaped LCGO/AgNWs composite (size of $20 \mathrm{~mm} \times 70 \mathrm{~mm} \times 3 \mu \mathrm{m}$ ) was measured by a digital multimeter (Model 187, Fluke Corporation, 
Washington, USA). The elastic properties were measured using a universal testing machine (UTM, INSTRON 5966, INSTRON, Massachusetts, USA).

\section{Fabrication of tactile sensor and resistance temperature detector}

The core silicone rubber dielectric was formed as a sheet by a conventional reaction of commercially available resins (Ecoflex 0030) in a square Petri dish. The next step in the fabricating process was cutting the resulting $\approx 1.5 \mathrm{~mm}$-thick silicone rubber film into a rectangular sheet, which serves as the dielectric of a capacitor. The typical dimensions of the non-strained dielectric film were $15 \mathrm{~mm} \times 15 \mathrm{~mm}$. The LCGO/AgNWs ink was separately printed on both sides of an asprepared dielectric film as a rectangular shape. The dimensions of the printed composite were 10 $\mathrm{mm} \times 10 \mathrm{~mm}$. All the electrodes of the tactile sensor were electrically connected to a $\mathrm{Cu}$ wire and the capacitance was measured with an LCR meter (LCR-914, Gwinstek, China). The conductive ink was printed on a sticker made of a PET film coated with a sticky pool on only one side and then electrically connected with $\mathrm{Cu}$ wire. The resistance change was measured by a digital multimeter. At the same time, the temperature change of the RTD sensor was measured by a digital thermometer (GT309, Giltron, Germany). 


\section{Conflict of interest}

There are no conflicts to declare.

\section{Supporting Information}

The optical image of LGGO/AgNWs ink, the self-healing mechanism, the electrical property of LCGO/AgNWs electrode..

\section{ORCID}

Seon Jeong Kim: 0000-0002-2867-6737

\section{Acknowledgement}

This work was supported by the Creative Research Initiative Center for Self-Powered Actuation in Korea. Additional support was from the Australian Research Council and the Australian National Fabrication Facility 


\section{REFERENCES}

[1] Rogers, J. A. Nature nanotechnology 2017, 12, 839-840.

[2] Kim, D.; Lu, N.; Kim, Y.; Kim, R.; Wang, S.; Wu, J.; Won, S. M.; Tao, H.; Islam, A.; Yu, K.

J.; Kim, T.; Chowdhury, R.; Ying, M.; Xu, L.; Li, M.; Chung, H.; Keum, H.; Mccormick, M.;

Liu, P.; Zhang, Y.; Omeenetto, F. G.; Huang, Y.; Coleman, T.; Rogers, J. A. science 2011, 333, 838-843.

[3] Kaltenbrunner, M.; Sekitani, T.; Reeder, J.; Yokota, T.; Kuribara, K.; Drack, M.;

Schwodiauer, R.; Graz, I.; Bauer-gogonea, S.; Bauer, S.; Someya, T. nature 2013, 499, 458-465.

[4] Lei, Z.; Wang, Q.; Sun, S.; Zhu, W.; Wu, P. Adv. Mater. 2017, 1700321, 1-6.

[5] Han, L.; Lu, X.; Wang, M.; Gan, D.; Deng, W.; Wang, K.; Fang, L.; Liu, K.; Chan, C. W.;

Tang, Y.; Weng, L.; Yuan, H. small 2017, 1601916, 1-9.

[6] Huynh, T.; Haick, H. Adv. Mater. 2016, 28, 138-143

[7] Darabi, M. A.; Khosrozadeh, A.; Mbeleck, R.; Liu, Y.; Chang, Q.; Jiang, J.; Cai, J.; Wang,

Q.; Luo, G.; Xing, M. Adv. Mater. 2017, 1700533, 1-8.

[8] Chi, W.; Wang, M.; Ma, C.; Wang, Y.; Li, X.; Yu, G. Nano lett. 2015, 15, 6276-6281.

[9] Odam, A. A.; Chayanupatkul, S.; Blaiszik, B. J.; Zhao, O.; Jackson, A. C.; Braun, P. V.;

Sottos, N. R.; White, S. R.; Moore, J. S. Adv. Mater. 2012, 24, 2578- 2581.

[10] Huynh, T.; Sonar, P.; Haick, H. Adv. Mater. 2017, 1604973, 1-14.

[11] Bandodkar, A. J.; Lopez, C. S.; Mohan, A. M. V.; Yin, L.; Kumar, R.; Wang, J. Sci. adv. 2016, $1601465,1-10$.

[12] Tee, B. C.; Wang, C.; Allen, R.; Bao, Z. Nature nanotechnology 2012, 7, 825-832.

[13] Guo, K.; Zhang, D.; Zhang, X.; Zhang, J.; Ding, L.; Li, B.; Zhang, S. Angew. Chem. 2015, $127,23395-12301$. 
[14] Li, Y.; Chen, S.; Wu, M.; Sun, J. Adv. Mater. 2012, 45, 4578-4582

[15] Wang, S.; Liu, N.; Su, J.; Li, L.; Long, F.; Zou, Z.; Jiang, X.; Gao, Y. ACS Nano 2017, 11, 2066-2074.

[16] Wang, H.; Zhu, D.; Jiang, W.; Wang, Y.; Leow, W. R.; Wang, H.; Chen, X. Adv.

Mater.2014, 26, 3638-3643.

[17] Chen, D.; Wang, D.; Yang, Y.; Huang, Q.; Zhu, S.; Zheng, Z. Adv. Energy. Mater. 2017, $1700890,1-23$.

[18] Bandodkar, A. J.; Mohan, V.; Lopez, C. S.; Ramires, J.; Wang, J. Adv. Electron. Mater. 2015, 1500289, 1-5.

[19] Jalili, R.; Aboutalebi, S. H.; Esrafilzadeh, D.; Shepherd, R. L.; Chen, J.; aminorroayaYamini, S.; Konstatinov, K.; Minett, a. I.; Razal, J. M.; Wallace, G. G. Adv. Funct. Mater 2013, $23,5345-5354$.

[20] Liang, J.; Li, L.; Tong, K.; Ren, Z.; Hu, W.; Niu, X.; Chen, Y.; Pei, Q. ACS Nano 2014, 8, 1590-1600.

[21] Cheng, H.; Huang, Y.; Cheng, Q.; Shi, G.; Jiang, L.; Qu, L. Adv. Funct. Mater. 2017, $1703096,1-8$ 
a

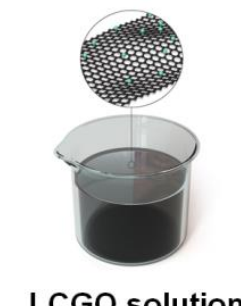

LCGO solution

$\downarrow$

AgNWs addition

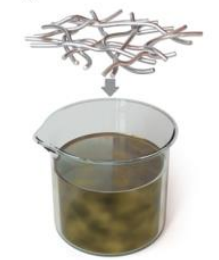

Mixing

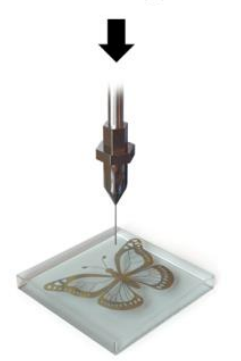

Printing b

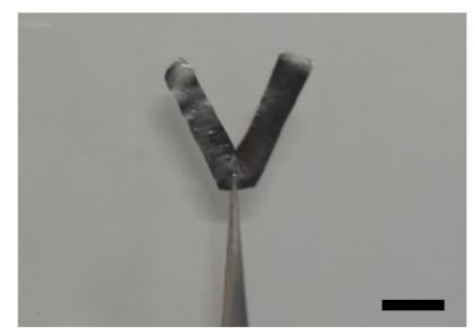

d

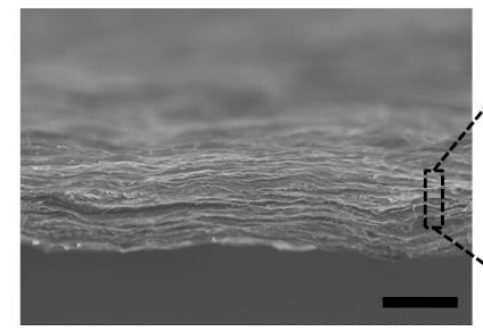

f

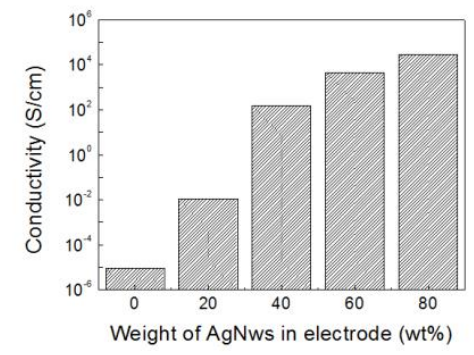

C

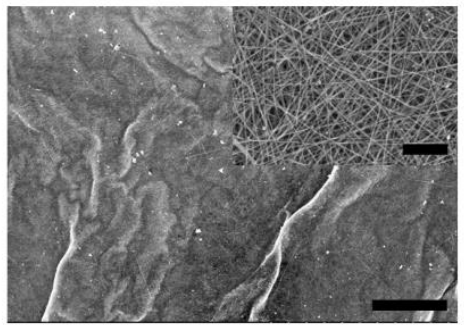

e

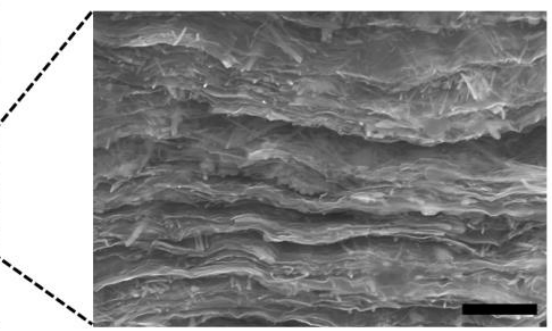

g

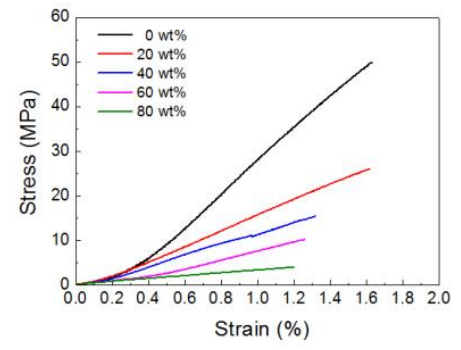

Figure 1. (a) The fabrication process for the self-healing electrode. The LCGO solution was mixed with AgNWs solution for the highly conductive self-healing electrode. The mixed solution has high viscosity and can be printed as butterfly-like shapes. (b) The photonic image of freestanding and flexible LCGO/AgNWs composite (scale bar: $1 \mathrm{~cm}$ ). (c) SEM image showing the surface morphology of the composite (scale bar: $20 \mu \mathrm{m}$ ). Inset image showing magnified SEM image (scale bar: $2 \mu \mathrm{m}$ ). (d) Cross-sectional SEM image showing the ultrathin thickness of the composite of about $3 \mu \mathrm{m}$. (scale bar: $2 \mu \mathrm{m}$ ) (e) Magnified SEM image showing the layered structure with LCGO and AgNWs (scale bar: $1 \mu \mathrm{m}$ ). The dependence of (f) electrical conductivity and (g) strainstress curve of LCGO/AgNWs composite on the weight of AgNWs. 

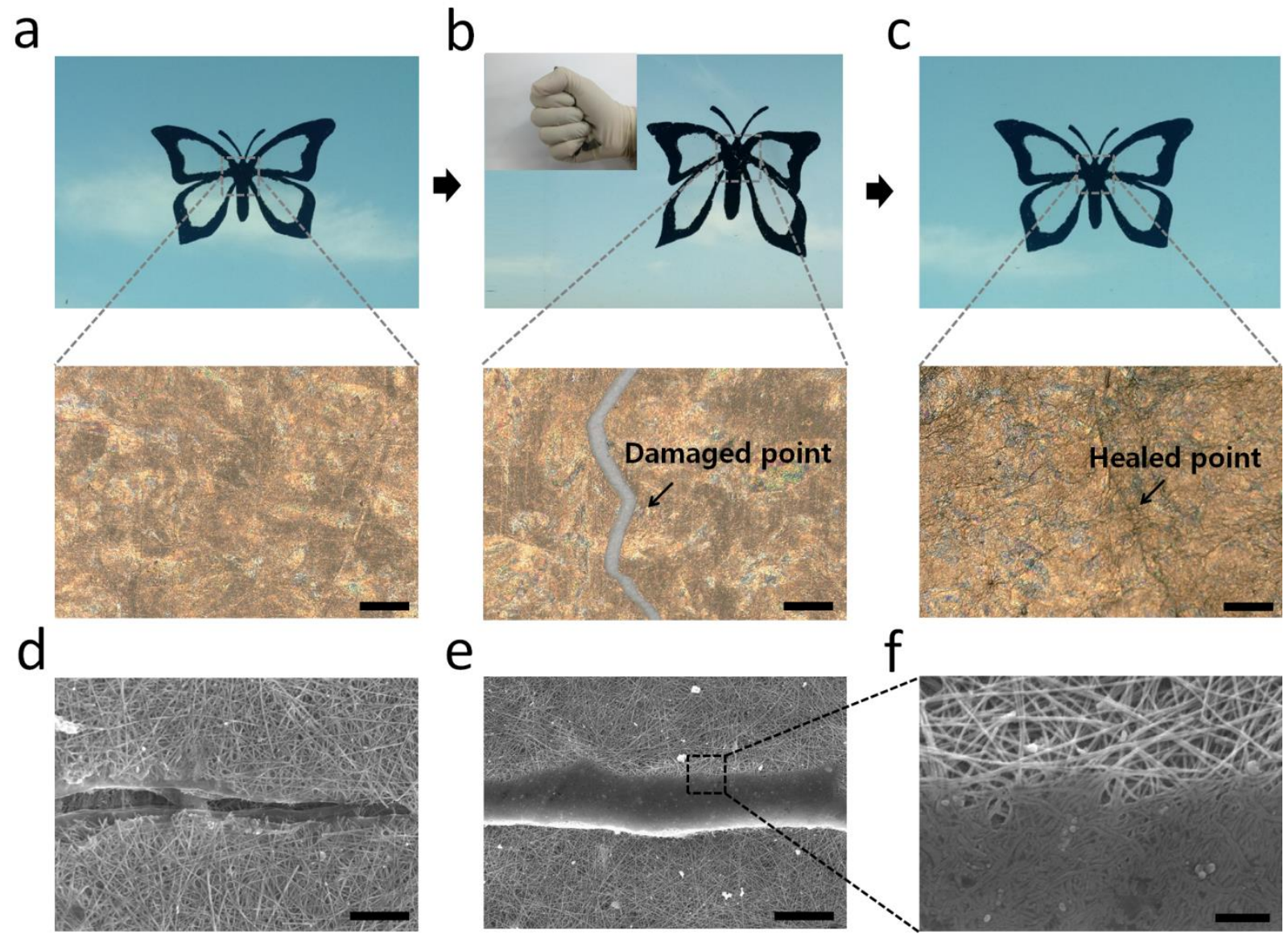

Figure 1. $(\mathrm{a}-\mathrm{c})$ The butterfly-shaped LCGO/AgNWs electrode was printed and was detached from the substrate in a freestanding state. The electrode was crumpled and damaged. The damaged point was recovered to the initial state after spraying with moisture. (scale bar: $1 \mathrm{~mm}$ ). (d) SEM image showing a microscale crack of the electrode (scale bar: $2 \mu \mathrm{m}$ ). (e) SEM image (scale bar: $2 \mu \mathrm{m}$ ) and (f) magnified SEM image (scale bar: $0.5 \mu \mathrm{m}$ ) showing the recovered point after the healing process. 
a

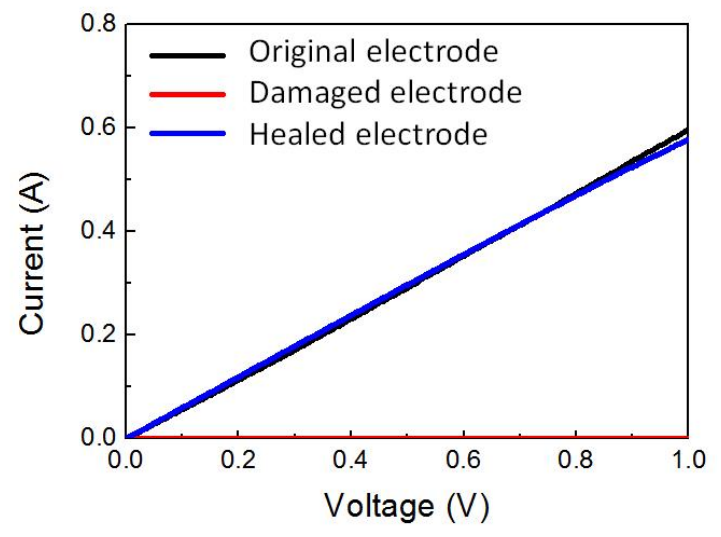

C

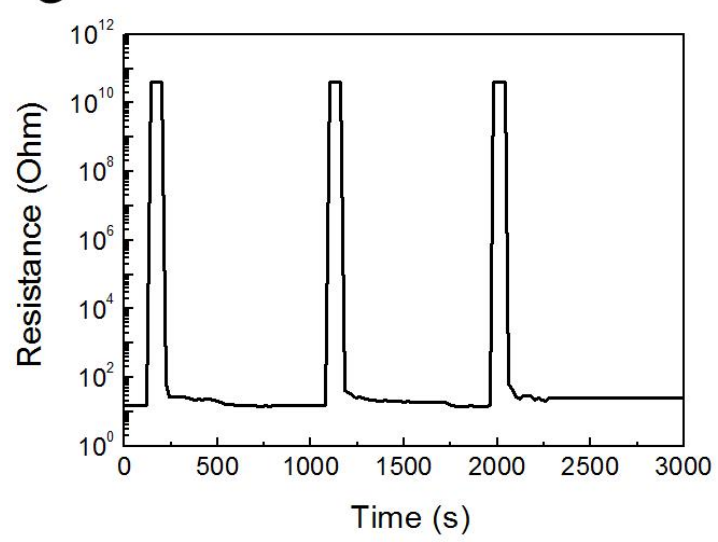

b

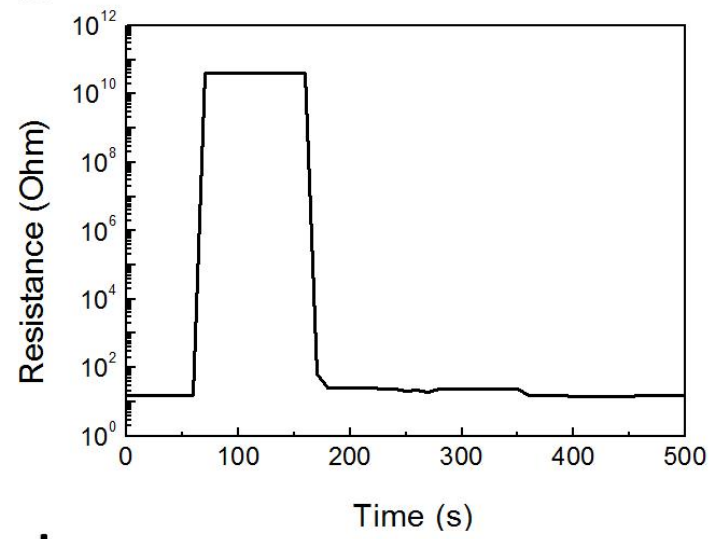

d

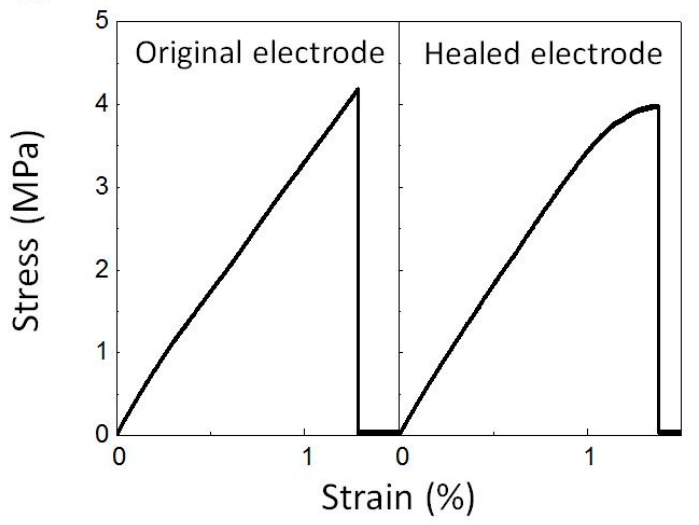

e
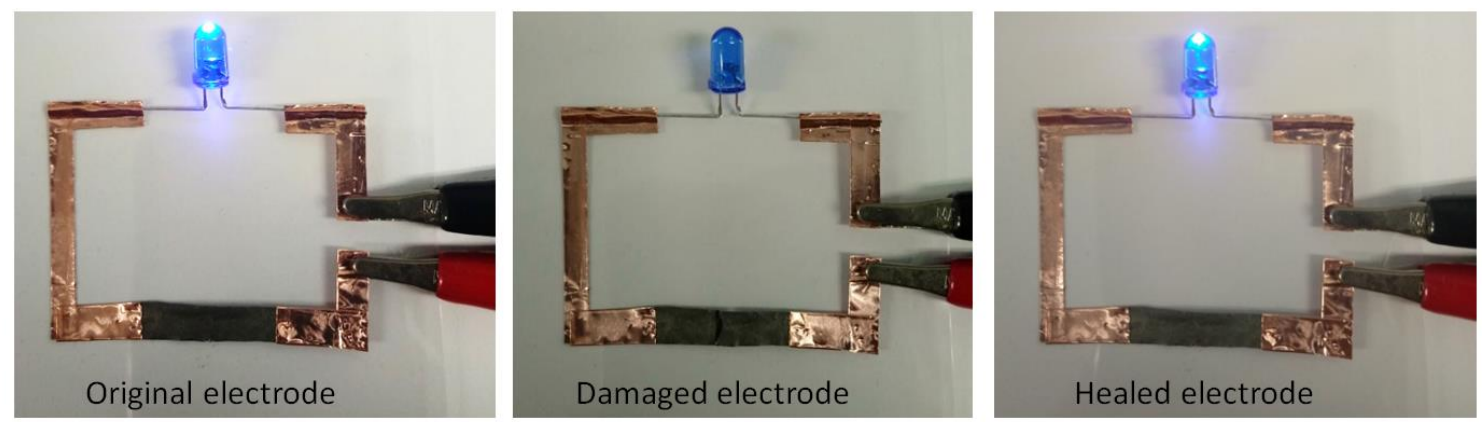

Figure 2. (a) Current-voltage curve using LCGO/AgNWs electrode. Original composite (black line), damaged composite (red line) and composite healed by moisture (blue line). (b) Time evolution of the electrical healing process using resistance measurement for a time of $500 \mathrm{~s}$ at room temperature. (c) Repeated healing process for three cuts at the same location. (d) Strain-stress 
curves of original and healed electrode. (e) Photographs of the electrode within a circuit with an LED before and after breaking, and after healing. 
a

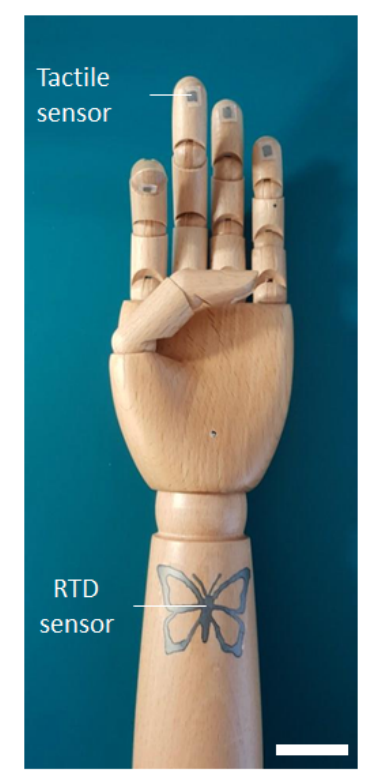

b

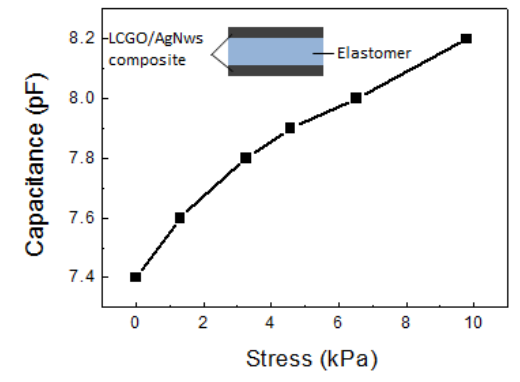

d

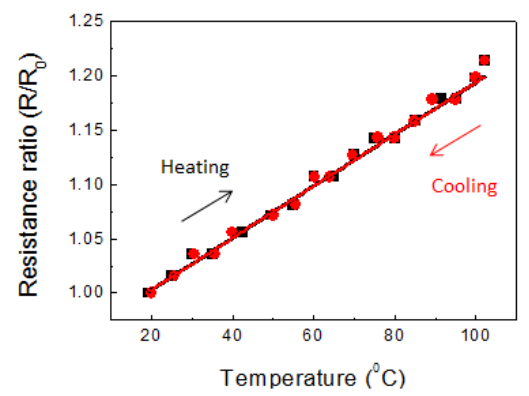

C

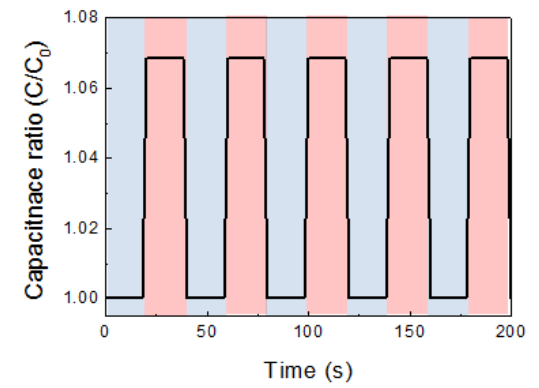

e

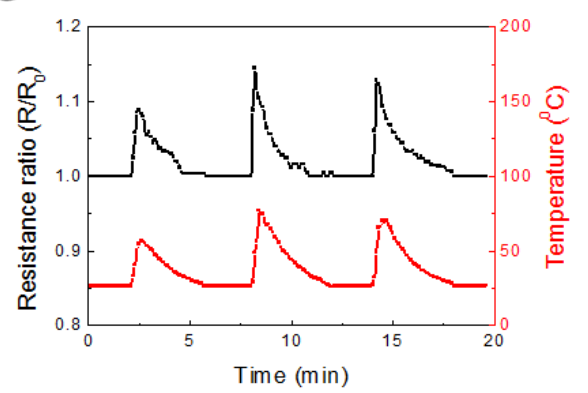

Figure 3. Application of self-healing artificial electronic skin. (a) The optical image of artificial electronic skin. The tactile sensor and resistance temperature detector were located on the fingers and wrist, respectively (scale bar: $3 \mathrm{~cm}$ ). (b) The capacitance of the tactile sensor with compression. (c) Capacitive response cycles during repeated compression (red) and releasing (blue). (d) The resistance variation with temperature. (e) Te resistance variation with time when the temperature was immediately changed. 
Graphic for manuscript (Table of Content)

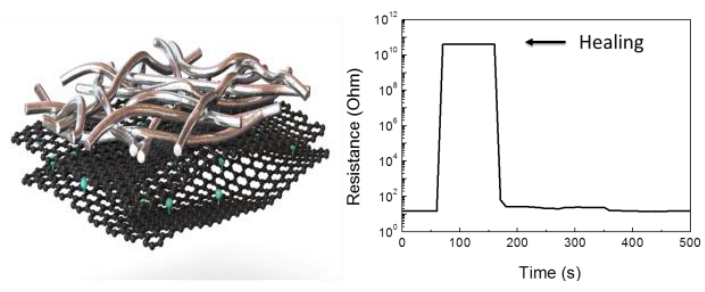

